DPUR/TH/44

March, 2015

\title{
Hawking Radiation of a Charged Black Hole in Quantum Gravity
}

\author{
Ichiro Oda $^{1}$ \\ Department of Physics, Faculty of Science, University of the Ryukyus, \\ Nishihara, Okinawa 903-0213, Japan.
}

\begin{abstract}
We study black hole radiation of a Reissner-Nordstrom black hole with an electric charge in the framework of quantum gravity. Based on a canonical quantization for a spherically symmetric geometry, under physically plausible assumptions, we solve the Wheeler-De Witt equation in the regions not only between the outer apparent horizon and the spatial infinity but also between the spacetime singularity and the inner apparent horizon, and then show that the mass loss rate of an evaporating black hole due to thermal radiation agrees with the semiclassical result when we choose an integration constant properly by physical reasoning. Furthermore, we also solve the Wheeler-De Witt equation in the region between the inner Cauchy horizon and the outer apparent horizon, and show that the mass loss rate of an evaporating black hole has the same expression. The present study is the natural generalization of the case of a Schwarzschild black hole to that of a charged Reissner-Nordstrom black hole.
\end{abstract}

\footnotetext{
${ }^{1}$ E-mail address: ioda@phys.u-ryukyu.ac.jp
} 


\section{Introduction}

Black holes have been thus far a quite fascinating research field from various points of view. On the one hand, from the observational viewpoint, the black holes are a very important constituent of the universe. It is nowdays believed that we have super-massive black holes with masses ranging from a million to a billion solar masses at the centers of many of galaxies including our Milky Way galaxy. Much smaller black holes could be also formed at the beginning of the universe, but might be evaporated away up to now [1].

From the theoretical point of view, black holes provide an intriguing arena to explore the challenges posed by the reconciliation between general relativity and quantum field theory. When enough energy density is concentrated in a small region in space, it naturally collapses to form a black hole. Thus, in the high energy regime, an elementary particle metamorphoses into a black hole. However, there is a sharp contradiction between black holes and elemetary particles. For instance, the density of states in gravity, which is deduced by the Bekenstein-Hawking entropy formula, is different from the density of states in any renormalizable quantum field theory [2]. To put differently, at the Planck scale, any quantum field theory reduces to a conformal field theory in the same spacetime dimension whereas the Bekenstein-Hawking entropy formula for black holes implies that gravity does to a conformal field theory in one less spacetime dimension [3]. This observation has culminated in success of the AdS/CFT correspondence [4].

A quantum-mechanical treatment of matter in the background of a classical black hole has indicated that the black hole radiates as a black body with a certain Hawking temperature [1]. Since the black hole radiation involves a mixture of gravity and quantum mechanics, the study of black holes seems to lead us into the territory of quatum gravity, which is the missing link in a complete picture of the fundamental forces in nature.

In our recent study [5], based on a canonical formalism for a spherically symmetric geometry $[6,7]$, we have considered the black hole radiation of a Schwarzshild black hole both inside and outside the apparent horizon in quantum gravity where it was shown that the mass loss rate due to the black hole radiation is equal to that evaluated by Hawking in the semiclassical approximation [8, 7]. ${ }^{2}$ Then, it is natural to ask ourselves whether our formulation can be extended to a charged black hole, that is, the Reissner-Nordstrom black hole, or not. In this article, we will present the affirmative answer to this question and show that the mass loss rate of an evaporating black hole with an electric charge also coincides with the result obtained in the semiclassical approach as in a Schwarzschild black hole.

This article is organized as follows: In the next section, on the basis of the canonical formalism of a system with a spherically symmetric black hole, which holds in the region bounded by the outer horizon and the spatial infinity or in the region bounded by the spacetime singularity and the inner Cauchy horizon, we calculate the mass loss rate due to black hole radiation. In Section 3, we do the same job for the region bounded by the inner and apparent horizons. The final section is devoted to a conclusion.

\footnotetext{
${ }^{2}$ See also related works $[9,10]$.
} 


\section{Formalism}

The four-dimensional action with which we start takes the following form: ${ }^{3}$

$$
S=\int d^{4} x \sqrt{-{ }^{(4)} g}\left[\frac{1}{16 \pi}{ }^{(4)} R-\frac{1}{4 \pi}{ }^{(4)} g^{\mu \nu}\left(D_{\mu} \Phi\right)^{\dagger} D_{\nu} \Phi-\frac{1}{16 \pi} F_{\mu \nu} F^{\mu \nu}\right],
$$

where $\Phi$ is a complex scalar field and its covariant derivative is given by

$$
D_{\mu} \Phi=\partial_{\mu} \Phi+i e A_{\mu} \Phi
$$

with $e$ and $A_{\mu}$ being the electric charge of $\Phi$ and the $U(1)$ gauge field, respectively. Moreover, $F_{\mu \nu}$ is the field strength defined as

$$
F_{\mu \nu}=\partial_{\mu} A_{\nu}-\partial_{\nu} A_{\mu}
$$

To clarify the four-dimensional meaning we put the suffix (4) in front of the metric tensor and the scalar curvature. As a final note, the Greek indices $\mu, \nu, \cdots$ take the four-dimensional values $0,1,2$ and 3 whereas the Latin ones $a, b, \cdots$ do the two-dimensional values 0 and 1 . Of course, it is straightforward to include the other matter fields as well as the cosmological constant in the action (1) even if we limit ourselves to the action for simplicity.

The most general spherically symmetric ansatz for the four-dimensional line element is of form

$$
\begin{aligned}
{ }^{(4)} d s^{2} & ={ }^{(4)} g_{\mu \nu} d x^{\mu} d x^{\nu} \\
& =g_{a b} d x^{a} d x^{b}+\phi^{2}\left(d \theta^{2}+\sin ^{2} \theta d \varphi^{2}\right),
\end{aligned}
$$

where the two-dimensional metric $g_{a b}$ and the radial function $\phi$ are the functions of only the two-dimensional coordinates $x^{a}$. The substitution of the ansatz (4) into the action (1) and then integration over the angular variables $(\theta, \varphi)$ produces the two-dimensional effective action

$$
\begin{aligned}
S & =\frac{1}{2} \int d^{2} x \sqrt{-g}\left[1+g^{a b} \partial_{a} \phi \partial_{b} \phi+\frac{1}{2} R \phi^{2}\right] \\
& -\int d^{2} x \sqrt{-g}\left[\phi^{2} g^{a b}\left(D_{a} \Phi\right)^{\dagger} D_{b} \Phi+\frac{1}{4} \phi^{2} F_{a b} F^{a b}\right],
\end{aligned}
$$

where we have assumed that $A_{a}$ and $\Phi$ are also the functions of the two-dimensional coordinates $x^{a}$ and set $A_{\theta}=A_{\varphi}=0$.

Next let us rewrite the action (5) in the form of a linear combination of the first-class constraints. Since in this section we wish to consider the region bounded by the outer apparent

\footnotetext{
${ }^{3}$ We mainly follow notation and conventions by Misner et al.'s textbook [11], for instance, the flat Minkowski metric $\eta_{\mu \nu}=\operatorname{diag}(-,+,+,+)$, the Riemann curvature tensor $R_{\nu \alpha \beta}^{\mu}=\partial_{\alpha} \Gamma_{\nu \beta}^{\mu}-\partial_{\beta} \Gamma_{\nu \alpha}^{\mu}+\Gamma_{\sigma \alpha}^{\mu} \Gamma_{\nu \beta}^{\sigma}-\Gamma_{\sigma \beta}^{\mu} \Gamma_{\nu \alpha}^{\sigma}$, and the Ricci tensor $R_{\mu \nu}=R^{\alpha}{ }_{\mu \alpha \nu}$. Throughout this article, we adopt the natural units $c=\hbar=G=1$. In this units, all quantities become dimensionless.
} 
horizon and the spatial infinity, or the region bounded by the spacetime singularity and the inner Cauchy apparent horizon, as usual we regard the $x^{0}$ coordinate as time to cover the region by spacelike hypersurfaces. Then, the appropriate ADM splitting of (1+1)-dimensional spacetime is given by $[6]$

$$
g_{a b}=\left(\begin{array}{cc}
\frac{\beta^{2}}{\gamma}-\alpha^{2} & \beta \\
\beta & \gamma
\end{array}\right)
$$

The normal unit vector $n^{a}$ which is orthogonal to the hypersurfaces $x^{0}=$ const reads

$$
n^{a}=\left(\frac{1}{\alpha},-\frac{\beta}{\alpha \gamma}\right) \text {. }
$$

The trace of the extrinsic curvature and the scalar curvature are given by

$$
\begin{aligned}
K & =\frac{\dot{\gamma}}{2 \alpha \gamma}-\frac{\beta^{\prime}}{\alpha \gamma}+\frac{\beta}{2 \alpha \gamma^{2}} \gamma^{\prime} \\
R & =2 n^{a} \partial_{a} K+2 K^{2}-\frac{2}{\alpha \sqrt{\gamma}} \partial_{1}\left(\frac{\alpha^{\prime}}{\sqrt{\gamma}}\right)
\end{aligned}
$$

where $\frac{\partial}{\partial x^{0}}=\partial_{0}$ and $\frac{\partial}{\partial x^{1}}=\partial_{1}$ are also denoted as an overdot and a prime, respectively.

With the help of these equations, one can cast the action (5) to the form

$$
\begin{aligned}
S & \equiv \int d^{2} x L \\
& =\int d^{2} x\left[\frac{1}{2} \alpha \sqrt{\gamma}\left\{1-\left(n^{a} \partial_{a} \phi\right)^{2}+\frac{1}{\gamma}\left(\phi^{\prime}\right)^{2}-K n^{a} \partial_{a}\left(\phi^{2}\right)+\frac{\alpha^{\prime}}{\alpha \gamma} \partial_{1}\left(\phi^{2}\right)\right\}\right. \\
& \left.+\alpha \sqrt{\gamma} \phi^{2}\left\{\left|n^{a} D_{a} \Phi\right|^{2}-\frac{1}{\gamma}\left|D_{1} \Phi\right|^{2}\right\}+\frac{1}{2} \alpha \sqrt{\gamma} \phi^{2} E^{2}\right] \\
& +\int d^{2} x\left[\frac{1}{2} \partial_{a}\left(\alpha \sqrt{\gamma} K n^{a} \phi^{2}\right)-\frac{1}{2} \partial_{1}\left(\frac{\alpha^{\prime}}{\sqrt{\gamma}} \phi^{2}\right)\right],
\end{aligned}
$$

where we have defined $E$ as

$$
E=\frac{1}{\sqrt{-g}} F_{01}=\frac{1}{\alpha \sqrt{\gamma}}\left(\dot{A}_{1}-A_{0}^{\prime}\right)
$$

Now the differentiation of the action (9) with respect to the time derivative of the canonical variables $\Phi\left(\Phi^{\dagger}\right), \phi, \gamma$ and $A_{1}$ leads to the corresponding canonical conjugate momenta $\pi_{\Phi}\left(\pi_{\Phi^{\dagger}}\right), \pi_{\phi}, \pi_{\gamma}$ and $\pi_{A}$ as follows:

$$
\begin{aligned}
& \pi_{\Phi}=\sqrt{\gamma} \phi^{2} n^{a}\left(D_{a} \Phi\right)^{\dagger}, \quad \pi_{\Phi^{\dagger}}=\sqrt{\gamma} \phi^{2} n^{a} D_{a} \Phi, \quad \pi_{\phi}=-\sqrt{\gamma} n^{a} \partial_{a} \phi-\sqrt{\gamma} K \phi \\
& \pi_{\gamma}=-\frac{1}{4 \sqrt{\gamma}} n^{a} \partial_{a}\left(\phi^{2}\right), \quad \pi_{A}=\phi^{2} E .
\end{aligned}
$$


Then, the Hamiltonian, which is defined as

$$
H=\int d x^{1}\left(\pi_{\Phi} \dot{\Phi}+\pi_{\Phi^{\dagger}} \dot{\Phi}^{\dagger}+\pi_{\phi} \dot{\phi}+\pi_{\gamma} \dot{\gamma}+\pi_{A} \dot{A}_{1}-L\right)
$$

is expressed in terms of a linear combination of constraints as expected from diffeomorphism invariance

$$
H=\int d x^{1}\left(\alpha H_{0}+\beta H_{1}+A_{0} H_{2}\right)
$$

where $\alpha, \beta$ and $A_{0}$ are non-dynamical Lagrange multiplier fields, and the Hamiltonian constraint, the momentum one and the constraint associated with the $U(1)$ gauge transformation are respectively given by

$$
\begin{aligned}
H_{0} & =\frac{1}{\sqrt{\gamma} \phi^{2}} \pi_{\Phi} \pi_{\Phi^{\dagger}}-\frac{\sqrt{\gamma}}{2}-\frac{\left(\phi^{\prime}\right)^{2}}{2 \sqrt{\gamma}}+\partial_{1}\left(\frac{\partial_{1}\left(\phi^{2}\right)}{2 \sqrt{\gamma}}\right)+\frac{\phi^{2}}{\sqrt{\gamma}}\left|D_{1} \Phi\right|^{2} \\
& -\frac{2 \sqrt{\gamma}}{\phi} \pi_{\phi} \pi_{\gamma}+\frac{2 \gamma \sqrt{\gamma}}{\phi^{2}} \pi_{\gamma}^{2}+\frac{\sqrt{\gamma}}{2 \phi^{2}} \pi_{A}^{2}, \\
H_{1} & =\frac{1}{\gamma}\left[\pi_{\Phi} D_{1} \Phi+\pi_{\Phi^{\dagger}}\left(D_{1} \Phi\right)^{\dagger}\right]+\frac{1}{\gamma} \pi_{\phi} \phi^{\prime}-2 \pi_{\gamma}^{\prime}-\frac{1}{\gamma} \pi_{\gamma} \gamma^{\prime}, \\
H_{2} & =-i e\left(\pi_{\Phi} \Phi-\pi_{\Phi^{\dagger}} \Phi^{\dagger}\right)-\pi_{A}^{\prime} .
\end{aligned}
$$

We are now in a position to apply this canonical formalism for understanding the Hawking radiation of a charged black hole in quantum gravity. We make use of the ingoing Vaidya metric [12] to describe a dynamical black hole. We therefore define the two-dimensional coordinates $x^{a}$ as

$$
x^{a}=\left(x^{0}, x^{1}\right)=(v-r, r),
$$

where $v$ is the advanced time coordinate. Next, let us fix the two-dimensional diffeomorphisms by the gauge conditions

$$
g_{a b}=\left(\begin{array}{cc}
\frac{\beta^{2}}{\gamma}-\alpha^{2} & \beta \\
\beta & \gamma
\end{array}\right)=\left(\begin{array}{cc}
-\left(1-\frac{2 M}{r}+\frac{Q^{2}}{r^{2}}\right) & \frac{2 M}{r}-\frac{Q^{2}}{r^{2}} \\
\frac{2 M}{r}-\frac{Q^{2}}{r^{2}} & 1+\frac{2 M}{r}-\frac{Q^{2}}{r^{2}}
\end{array}\right),
$$

where $M=M\left(x^{0}, x^{1}\right)$ and $Q=Q\left(x^{0}, x^{1}\right)$ play the role of the mass function and the electric charge function of the charged black hole, respectively. From the gauge conditions (16), the two-dimensional line element takes the form of the Vaidya metric [12]

$$
\begin{aligned}
d s^{2} & =g_{a b} d x^{a} d x^{b} \\
& =-\left(1-\frac{2 M}{r}+\frac{Q^{2}}{r^{2}}\right) d v^{2}+2 d v d r .
\end{aligned}
$$

Furthermore, for the $U(1)$ gauge transformation, we shall take the gauge condition

$$
A_{0}=A_{1}=\frac{Q}{r}
$$


which becomes

$$
A_{v}=\frac{Q}{r}, \quad A_{r}=0
$$

where we have used the definition (15).

For a dynamical black hole, we make use of the local definition of the horizon, namely, the apparent horizon, rather than the global one, the event horizon. Note that in a charged black hole there are two apparent horizons which are defined as

$$
r_{ \pm}=M \pm \sqrt{M^{2}-Q^{2}}
$$

where $r_{+}$and $r_{-}$are respectively the outer and the inner horizons. In the extremal black hole $M=Q$, the two horizons coalesce into one horizon. For simplicity, in this article we will confine ourselves to the non-extremal black hole $M \neq Q$ since the extremal case can be treated in a similar way even if we will later use the property that the Hawking temperature of the extremal black hole is zero.

Since we take account of a massless scalar field which follows along the null geodesics, it seems to be physically reasonable to assume that the scalar field $\Phi$ depends on only the null coordinate $v$, by which the mass function $M$ and the charge function $Q$ also become the function of the advanced time coordinate $v$. Furthermore, the radial function $\phi$ is assumed to be a radial coordinate $r$. Thus, we take the following assumptions

$$
\Phi \approx \Phi(v), \quad M \approx M(v), \quad Q \approx Q(v), \quad \phi \approx r
$$

Henceforth, we shall use the simbol $\approx$ to indicate the equalities holding under the assumptions (21). Under the assumption $Q \approx Q(v)$, the gauge condition (18) (or (19)) gives rise to an electric field

$$
E \approx \frac{Q}{r^{2}}
$$

It will turn out that the assumptions (21) play a critical role in simplifying the diffeomorphism constraints and lead to a solvable model of a quantum black hole.

Now we will turn our attention to quantum theory. To do that, under the assumptions (21), using the gauge conditions (16) and (19), let us first simplify the canonical conjugate momenta (11)

$$
\begin{aligned}
\pi_{\Phi} & \approx \phi^{2}\left(\partial_{v} \Phi^{\dagger}-i e \frac{Q}{\phi} \Phi^{\dagger}\right), \quad \pi_{\Phi^{\dagger}} \approx \phi^{2}\left(\partial_{v} \Phi+i e \frac{Q}{\phi} \Phi\right) \\
\pi_{\phi} & \approx \frac{1}{1+\frac{2 M}{\phi}-\frac{Q^{2}}{\phi^{2}}}\left[\partial_{v} M-\frac{Q \partial_{v} Q}{\phi}+\frac{2 M^{2}}{\phi^{2}}+\frac{Q^{2}}{\phi^{2}}\left(1-\frac{M}{\phi}\right)\right], \\
\pi_{\gamma} & \approx \frac{1}{1+\frac{2 M}{\phi}-\frac{Q^{2}}{\phi^{2}}}\left(M-\frac{Q^{2}}{2 \phi}\right), \quad \pi_{A} \approx Q .
\end{aligned}
$$


Then, after some calculations, it turns out that the momentum constraint becomes identical with the Hamiltonian constraint up to an irrelevant overall factor

$$
\begin{aligned}
\sqrt{\gamma} H_{0} & \approx \gamma H_{1} \\
& \approx \frac{2}{\phi^{2}} \pi_{\Phi} \pi_{\Phi^{\dagger}}-\left(1+\frac{2 M}{\phi}-\frac{Q^{2}}{\phi^{2}}\right) \pi_{\phi}+\frac{2 M^{2}}{\phi^{2}}+\frac{Q^{2}}{\phi^{2}}\left(1-\frac{M}{\phi}\right)
\end{aligned}
$$

This compatibility between the momentum and the Hamiltonian constraints justifies the assumptions (21) in quantum gravity. The reduction of the dynamical degree of freedom associated with the radial function $\phi$ can be understood as a result of the momentum constraint. In addition to it, the first-class constraint coming from the $U(1)$ gauge transformation takes the form

$$
H_{2} \approx-i e\left(\pi_{\Phi} \Phi-\pi_{\Phi^{\dagger}} \Phi^{\dagger}\right)-\partial_{v} Q
$$

The constraint (24) as an operator equation on the wave functional $\Psi$ gives rise to the Wheeler-De Witt equation

$$
\left[-\frac{2}{\phi^{2}} \frac{\partial^{2}}{\partial \Phi \partial \Phi^{\dagger}}+i\left(1+\frac{2 M}{\phi}-\frac{Q^{2}}{\phi^{2}}\right) \frac{\partial}{\partial \phi}+\frac{2 M^{2}}{\phi^{2}}+\frac{Q^{2}}{\phi^{2}}\left(1-\frac{M}{\phi}\right)\right] \Psi=0 .
$$

Now it is easy to find a special solution of the Wheeler-De Witt equation (26) by the method of separation of variables. The result is given by

$$
\Psi=\left(B e^{\sqrt{A}\left(\Phi+\Phi^{\dagger}\right)}+C e^{-\sqrt{A}\left(\Phi+\Phi^{\dagger}\right)}\right) e^{i\left[M \log \phi+\frac{2 A-M^{2}-Q^{2}}{2 \sqrt{M^{2}+Q^{2}}} \log \frac{\phi-\omega_{-}}{\phi-\omega_{+}}-\frac{M}{2} \log \left(\phi-\omega_{+}\right)\left(\phi-\omega_{-}\right)\right]},
$$

where $A, B$ and $C$ are integration constants. We have defined $\omega_{ \pm}=-M \pm \sqrt{M^{2}+Q^{2}}$.

Next, one must impose the gauge constraint $H_{2}$ on the wave functional $\Psi$

$$
\left[e\left(\Phi^{\dagger} \frac{\partial}{\partial \Phi^{\dagger}}-\Phi \frac{\partial}{\partial \Phi}\right)-\partial_{v} Q\right] \Psi=0
$$

It is easy to check that when the solution (27) is substituted into this equation, Eq. (28) is satisfied only when

$$
\partial_{v} Q=0, \quad \Phi^{\dagger}=\Phi
$$

Alternatively, the second equation can be expressed as $e=0$. In other words, the matter field must be neutral. We will see later that Eq. (29) is consistent with the field equations.

Provided that the expectation value $\langle\mathcal{O}\rangle$ of an operator $\mathcal{O}$ is defined as

$$
<\mathcal{O}>=\frac{1}{\int d \Phi|\Psi|^{2}} \int d \Phi \Psi^{*} \mathcal{O} \Psi
$$


by using (23) or (24) one can calculate the expectation value of mass loss rate $<\partial_{v} M>$

$$
<\partial_{v} M>=-\frac{2 A}{\phi^{2}}
$$

In our previous article [5], it has been shown that in the case of a Schwarzschild black hole $(Q=0)$, this result precisely coincides with that obtained by Hawking in his semiclassical approach. It will be shown later that the result (31) also agrees with the semiclassical result of the mass loss rate of a charged black hole due to the Hawking radiation.

Here let us check whether the above assumptions (21) are compatible with the field equations. The field equations obtained from the action (5) are given by

$$
\begin{aligned}
& -\frac{2}{\phi} \nabla_{a} \nabla_{b} \phi+\frac{2}{\phi} g_{a b} \nabla_{c} \nabla^{c} \phi+\frac{1}{\phi^{2}} g_{a b} \partial_{c} \phi \partial^{c} \phi-\frac{1}{\phi^{2}} g_{a b} \\
= & 4\left[\left(D_{a} \Phi\right)^{\dagger} D_{b} \Phi-\frac{1}{2} g_{a b}\left(D_{c} \Phi\right)^{\dagger} D^{c} \Phi+\frac{1}{2} F_{a c} F_{b}{ }^{c}-\frac{1}{8} g_{a b} F_{c d}^{2}\right], \\
& \frac{1}{\sqrt{-g}} \partial_{a}\left(\sqrt{-g} g^{a b} \partial_{b} \phi\right)-\frac{1}{2} R \phi=-2 \phi\left(D_{a} \Phi\right)^{\dagger} D^{a} \Phi-\frac{1}{2} \phi F_{a b}^{2}, \\
& D_{a}\left(\sqrt{-g} \phi^{2} g^{a b} D_{b} \Phi\right)=0, \\
& \frac{1}{\sqrt{-g}} \partial_{b}\left(\sqrt{-g} \phi^{2} F^{a b}\right)=i e \phi^{2} g^{a b}\left[\Phi^{\dagger} D_{b} \Phi-\Phi\left(D_{b} \Phi\right)^{\dagger}\right] .
\end{aligned}
$$

The Vaidya metric (17) gives us the metric tensor

$$
g_{a b}=\left(\begin{array}{cc}
g_{v v} & g_{v r} \\
g_{r v} & g_{r r}
\end{array}\right)=\left(\begin{array}{cc}
-\left(1-\frac{2 M}{r}+\frac{Q^{2}}{r^{2}}\right) & 1 \\
1 & 0
\end{array}\right) .
$$

To check the compatibility of the assumptions (21) with the field equations (32), let us make an ansatz that the variables have the form

$$
M \approx M(v), \quad Q \approx Q(v), \quad \phi \approx r
$$

but the scalar field is the function of both $v$ and $r$, i.e., $\Phi \approx \Phi(v, r)$. The first field equation in (32) is satisfied if the following relations hold:

$$
\partial_{r} \Phi \approx 0, \quad \Phi^{\dagger} \approx \Phi, \quad \partial_{v} Q \approx 0, \quad \partial_{v} \Phi \approx \frac{1}{r} \sqrt{\frac{\partial_{v} M}{2}},
$$

where note that $\Phi^{\dagger}=\Phi$ means $e=0$ at the same time. Namely, the field equations require that $\Phi$ is a real field and the electric charge $Q$ is constant. This fact is consistent with Eq. (29).

Then, the second and last equations in (32) are satisfied automatically. Finally, using Eq. (35), the third field equation in (32) gives us the equation

$$
2 r \partial_{v} \partial_{r} \Phi+2 \partial_{v} \Phi+r\left(1-\frac{2 M}{r}+\frac{Q^{2}}{r^{2}}\right) \partial_{r}^{2} \Phi \approx 0 .
$$


This equation (36) together with Eq. (35) requires us to limit ourselves to working with the vicinity of the apparent horizons (20) [5]. In other words, for the consistency of the field equations, in addition to the assumptions (21), one has to supplement one more assumption

$$
r \approx r_{ \pm} \equiv M \pm \sqrt{M^{2}-Q^{2}}
$$

Consequently, the field equations become

$$
\begin{aligned}
\Phi^{\dagger} & \approx \Phi, \quad e \approx 0, \quad Q \approx \text { const. } \\
\partial_{r} \Phi & \approx 0, \\
\partial_{v} \Phi & \approx \frac{1}{r} \sqrt{\frac{\partial_{v} M}{2}} \approx \frac{1}{r_{ \pm}} \sqrt{\frac{\partial_{v} M}{2}} \\
\partial_{r} \partial_{v} \Phi & \approx \partial_{v} \partial_{r} \Phi \approx-\frac{1}{r^{2}} \sqrt{\frac{\partial_{v} M}{2}} \approx-\frac{1}{r_{ \pm}^{2}} \sqrt{\frac{\partial_{v} M}{2}}
\end{aligned}
$$

Then, the solution is found to be

$$
\Phi(v, r)=\left(1-\frac{2 M}{r}+\frac{Q^{2}}{r^{2}}\right)^{2} \frac{r_{ \pm}^{2}}{r_{ \pm}^{2}-Q^{2}} \frac{1}{4 \sqrt{2 \partial_{v} M}}+\int^{v} d v \frac{1}{r_{ \pm}} \sqrt{\frac{\partial_{v} M}{2}}
$$

As a result, we have

$$
\Phi(v, r) \approx \int^{v} d v \frac{1}{r_{ \pm}} \sqrt{\frac{\partial_{v} M}{2}}
$$

which means that one can set $\Phi(v, r) \approx \Phi(v)$ in the vicinity of the apparent horizons. In this sense, the assumptions (21), if the assumption (37) is added, are at least classically consistent with the field equations (32) as long as the matter field is electrically neutral and the black hole charge $Q$ is a constant.

\section{Black hole radiation in the region bounded between the inner and outer horizons}

In this section, we wish to consider the Hawking radiation in the region bounded between the inner and outer apparent horizons. A peculiar characteristic feature is that in this region the Killing vector $\frac{\partial}{\partial t}$ becomes spacelike, so that one must foliate this region with a family of spacelike hypersurfaces such as $r=$ const. We have already constructed such a canonical formalism reflecting this fact $[7,5]$ so we can apply it for the present purpose. The argument proceeds in a perfectly analogous way to the case treated in the previous section so let us explain the content in a concise manner. 
In this region, the ADM splitting of $(1+1)$-dimensional spacetime is of form $[7,5]$

$$
g_{a b}=\left(\begin{array}{cc}
\gamma & \beta \\
\beta & \frac{\beta^{2}}{\gamma}-\alpha^{2}
\end{array}\right)
$$

The normal unit vector $n^{a}$ to the Cauchy hypersurfaces $x^{0}=$ const reads

$$
n^{a}=\left(\frac{\beta}{\alpha \gamma},-\frac{1}{\alpha}\right) \text {. }
$$

The trace of the extrinsic curvature is calculated to be

$$
K=-\frac{\gamma^{\prime}}{2 \alpha \gamma}+\frac{\dot{\beta}}{\alpha \gamma}-\frac{\beta}{2 \alpha \gamma^{2}} \dot{\gamma}
$$

Then, the canonical conjugate momenta $p_{\Phi}, p_{\Phi^{\dagger}}, p_{\phi}, p_{\gamma}$ and $p_{A}$ are given by

$$
\begin{aligned}
p_{\Phi} & \approx-\phi^{2}\left(\partial_{v} \Phi^{\dagger}-i e \frac{Q}{\phi} \Phi^{\dagger}\right), \quad p_{\Phi^{\dagger}} \approx-\phi^{2}\left(\partial_{v} \Phi+i e \frac{Q}{\phi} \Phi\right), \\
p_{\phi} & \approx \frac{1}{1-\frac{2 M}{\phi}+\frac{Q^{2}}{\phi^{2}}} \partial_{v} M+1-\frac{M}{\phi}, \\
p_{\gamma} & \approx-\frac{\phi}{2}, \quad p_{A} \approx-Q,
\end{aligned}
$$

where we have used $Q=$ const. The momentum constraint turns out to become proportional to the Hamiltonian constraint again ${ }^{4}$

$$
\begin{aligned}
\sqrt{\gamma} H_{0} & \approx-\gamma H_{1} \\
& \approx \frac{2}{\phi^{2}} p_{\Phi} p_{\Phi^{\dagger}}-\left(1-\frac{2 M}{\phi}+\frac{Q^{2}}{\phi^{2}}\right)\left(p_{\phi}-1+\frac{M}{\phi}\right) .
\end{aligned}
$$

This compatibility between the momentum and the Hamiltonian constraints justifies the assumptions (21) in quantum gravity as well. The gauge constraint $H_{2}$ becomes vanishing when the matter field is real $\Phi^{\dagger}=\Phi$ and the black hole charge $Q$ is a constant.

An imposition of the constraint (45) as an operator equation on the wave functional $\Psi$ produces the Wheeler-De Witt equation

$$
\left[-\frac{2}{\phi^{2}} \frac{\partial^{2}}{\partial \Phi \partial \Phi^{\dagger}}-\left(1-\frac{2 M}{\phi}+\frac{Q^{2}}{\phi^{2}}\right)\left(-i \frac{\partial}{\partial \phi}-1+\frac{M}{\phi}\right)\right] \Psi=0 .
$$

Then, a special solution of the Wheeler-De Witt equation (46) is given by

$$
\Psi=\left(B e^{\sqrt{A}\left(\Phi+\Phi^{\dagger}\right)}+C e^{-\sqrt{A}\left(\Phi+\Phi^{\dagger}\right)}\right) e^{i\left[\phi-M \log \phi-\frac{A}{\sqrt{M^{2}-Q^{2}}} \log \frac{\phi-r_{+}}{\phi-r_{-}}\right]},
$$

\footnotetext{
${ }^{4}$ Precisely speaking, since we consider the real matter field, we should set $p_{\Phi}=p_{\Phi \dagger}$, but for a generality we do not do so since the obtained result is in essence unchanged.
} 
where $A, B$ and $C$ are integration constants. As before, the expectation value of mass loss rate $\left\langle\partial_{v} M>\right.$ is easily calculated to be

$$
<\partial_{v} M>=-\frac{2 A}{\phi^{2}}
$$

which is the same expression as Eq. (31). After the condition (37) is inserted to Eq. (48) (or Eq. (31)), we obtain

$$
<\partial_{v} M>=-\frac{2 A}{r_{ \pm}^{2}}
$$

This result holding in the region $r_{-}<r<r_{+}$precisely coincides with that in the region $0<r<r_{-}$or $r_{+}<r<\infty$.

Here there is an important caveat. It is well-known that the inner Cauchy horizon $r=r_{-}$is unstable and inflates rapidly under the metric perturbations, what is called, "mass inflation" $[13,14,15]$. Then, our formalism should be applied only for the outer apparent horizon $r=r_{+}$ since we assume the stability of the apparent horizons implicitly.

The remaining work is to fix the integration "constant" $A$ in the sense that $A$ is independent of $\Phi$ and $\phi$. In the present formalism, however, it is difficult to determine the vaule of $A$ in principle since the physical state $\Psi$ is not uniquely defined in the whole spacetime region and the norm $\int d \Phi|\Psi|^{2}$ is not finite so that we will rely on the physical reasoning.

First of all, let us recall that the mass loss rate of a charged black hole should vanish for the extremal black hole since the extremal black hole has the vanishing Hawking temperature because of the formula $T_{H} \propto r_{+}-r_{-}$for the charged black hole. Next, notice that since $A$ is a dimensionless "constant", it must take the form of $\left(\frac{r_{+}-r_{-}}{r_{+}}\right)^{n}$ where $n$ is a positive number. Note that the possibility of $\left(\frac{r_{+}-r_{-}}{r_{-}}\right)^{n}$ is excluded since it becomes divergent in the limit of $Q \rightarrow 0 .{ }^{5}$ Finally, Stefan's law determines $n=4$ since the energy flux for a massless field is proportional to $T_{H}^{4}$. Accordingly, we have

$$
A=c\left(\frac{r_{+}-r_{-}}{r_{+}}\right)^{4},
$$

where $c$ is some numerical constant. Hence, the expectation value of mass loss rate is given by

$$
\begin{aligned}
<\partial_{v} M> & =-2 c \frac{1}{\phi^{2}}\left(\frac{r_{+}-r_{-}}{r_{+}}\right)^{4} \\
& \approx-2 c \frac{\left(r_{+}-r_{-}\right)^{4}}{r_{+}^{6}}
\end{aligned}
$$

where we have used $\phi \approx r_{+}$as explained above. It is remarkable that this result for a charged black hole exactly coincides with the result obtained in the semiclassical approach up to an overall constant.

\footnotetext{
${ }^{5}$ Incidentally, in the case of a Schwarzschild black hole, there is only one apparent horizon, so the integration constant $A$ must be strictly a constant.
} 


\section{Conclusion}

In this article, we have derived the mass loss rate of a charged black hole in a purely quantummechanical way, but in order to fix the integration constant we have had to reply on the physical reasoning, for instance, the vanishing Hawking temperature for the extremal black hole. The difficulty of determining the integration constants such as $A$ is connected with the difficulty of determining the physical state uniquely which holds in the whole spacetime region and the norm is not well-defined. In spite of such a difficulty, it is surprising that the present formalism offers a much greater degree of flexibility in that it explains the the mass loss rate of not only a charged black hole but also a Schwarzschild black hole due to the Hawking radiation.

In a recent progress on black hole physics, many of important studies are closely related to the quantum-mechanical behavior of the (apparent or event) horizon of a black hole. In such

a situation, the present formalism seems to provide for a useful playground. Although there remain a lot of problems to be solved, for instance, understanding of the proper definition of the norm and the meaning of the physical state etc., the approach adopted in this article appears to deserve careful study in future.

\section{Acknowledgements}

This work is supported in part by the Grant-in-Aid for Scientific Research (C) No. 25400262 from the Japan Ministry of Education, Culture, Sports, Science and Technology.

\section{References}

[1] S. W. Hawking, Comm. Math. Phys. 43 (1975) 199.

[2] A. Shomer, arXiv:0709.3555 [hep-th].

[3] O. Aharony and T. Banks, JHEP 9903 (1999) 016, arXiv:hep-th/9812237.

[4] J. M. Maldacena, Adv. Theor. Math. Phys. 2 (1998) 231, arXiv:hep-th/9711200.

[5] I. Oda, arXiv:1503.02141 [hep-th].

[6] P. Hajicek, Phys. Rev. D 30 (1984) 1178.

[7] A. Hosoya and I. Oda, Prog. Theor. Phys. 97 (1997) 233.

[8] A. Tomimatsu, Phys. Lett. B 289 (1992) 283.

[9] A. Hosoya and I. Oda, gr-qc/9605069.

[10] I. Oda, Phys. Lett. B 409 (1997) 88; Phys. Rev. D 57 (1998) 2415. 
[11] C. W. Misner, K. S. Thorne and J. A. Wheeler, "Gravitation", W H Freeman and Co (Sd), 1973.

[12] P. C. Vaidya, Proc. Indian Acad. Sci. A 33 (1951) 264.

[13] E. Poisson and W. Israel, Phys. Rev. D 41 (1990) 1796.

[14] A. Ori, Phys. Rev. Lett. 67 (1991) 789; 68 (1992) 2117.

[15] P. R. Brady and C. M. Chambers, Phys. Rev. D 51 (1995) 4177; P. R. Brady and J. D. Smith, Phys. Rev. Lett. 75 (1995) 1256. 